Ann. Zootech., I97I, 20 (2), 25I-257.

\title{
INFLUENCE DE LA CASTRATION \\ ET DE LA NATURE DES GLUCIDES DE LA RATION SUR LA CROISSANGE ET LA QUALITÉ DES CARCASSES DE BOVINS ABATTUS A 24 MOIS
}

\author{
Y. GEAY et C. MALTERRE \\ avec Ia collaboration technique de G. CUYLLE \\ Station de Recherches sur l'Élevage des Ruminants, \\ Centre de Recherches de Clermont-Ferrand, I. N. R. A., \\ 63 - Saint-Genès-Champanelle
}

\section{INTRODUCTION}

De nombreux auteurs (Richter et al., I960 $a$ et $b$ - I96i - KLosterman et al., I954 - et plus récemment $B R \ddot{R A N A ̈ N G}$, 1969) ont montré que la castration des bovins mâles diminue le gain de poids vif, ainsi que l'efficacité alimentaire et accroît la vitesse d'engraissement. De l'ensemble des résultats très variables des 56 expériences étrangères passées en revue par TurTon (I969), il ressort que dans des conditions d'élevage et d'alimentation identiques, les taurillons abattus entre $\mathrm{I} 2$ et $\mathrm{r} 8$ mois produisent des carcasses plus lourdes comportant davantage de muscles et fournissent de la viande dont les qualités organoleptiques sont aussi bonnes que celles des bouvillons de même âge. Aussi la production de viande de jeunes bovins, actuellement en pleine extension, ne se fait-elle en France qu'à partir d'animaux non castrés.

En revanche, la production de viande à partir de taureaux engraissés entre 20 et 24 mois est encore très peu répandue par suite des problèmes posés par la conduite des animaux et la qualité de la viande produite. Aucune étude, à notre connaissance, n'a montré jusqu'ici l'intérêt qu'elle peut cependant présenter. Par ailleurs, la composition du régime peut influer sur le gain de poids vif et la composition des carcasses, et les différences de vitesse d'engraissement entre animaux de diverses races peuvent être modifiées par le régime alimentaire (Y. GEAY, C. Béranger, 1969).

Aussi avons-nous cherché à préciser dans quelle mesure les différences de précocité et de gain de poids vif dues à la castration (si elles persistent jusqu'à 24 mois) peuvent être réduites sous l'influence de la nature de la ration. 


\section{MATÉRIEL E'T MÉTHODES}

Selon un dispositif factoriel, nous avons comparé, entre 20 ct 24 mois les performances d'engraissement de 20 boufs à celles de 20 taureaux recevant deux régimes isoénergétiques : une même proportion de l'énergic nette de la ration étant fournie soit par du saccharose, soit par l'amidon - certaines observations pratiques permettant en effet de penser que les betteraves favorisaient moins l'engraissement que les céréales.

Quarante veaux de race salers, âgés de ıo mois, ont été répartis en deux groupes de vingt animaux : ceux du premier groupe pesant $289 \mathrm{~kg}$ en moyenne ont été castrés, ceux du second groupe d'un poids moyen de $284 \mathrm{~kg}$ ne l'ont pas été. Jusqu'à 20 mois, l'ensemble des animaux a été conduit de façon identique : à l'auge durant l'hiver, puis au pâturage pendant la période d'été. Entre ro et 20 mois, les gains de poids vif n'ont pas été suffisamment élevés pour mettre en évidence une influence significative de la castration. En effet, l'ensemble des animaux n'a reçu au cours de la période hivcrnale qu'un foin de qualité moyenne (5 à $6 \mathrm{~kg}$ de matière sèche par animal et par jour et $500 \mathrm{~g}$ de concentré leur assurant un croît de $400-500 \mathrm{~g} / \mathrm{j}$. En été, au pâturage sur prairies naturelles de montagne, ils ont eu un croît de $800-900 \mathrm{~g} / \mathrm{j}$ qui est normal pour des animaux de cet âge ne recevant pas de céréales en supplément (C. BÉranger, M. Petit, i97o).

A l'âge de 20 mois, nous avons constitué ro couples de bœufs et ro couples de taurcaux. Un animal de chaque couple a reçu $3 \mathrm{~kg}$ de foin de luzerne condensé (broyé à la grille de $3 \mathrm{~mm}$ et aggloméré) et des betteraves fourragères à raison de $6 \mathrm{p}$. 100 du poids vif ; son homologue a reçu la même quantité de foin de luzerne condensé et de l'orge apportant la même quantité d'énergie nette (exprimée en unités fourragères) que celle fournie par les betteraves. Nous avons pour cela attribué aux betteraves la valeur énergétique nette de $0,9 \mathrm{UF} / \mathrm{kg}$ de matière sèche et à l'orge celle de $\mathrm{I}, \mathrm{I}_{4} \mathrm{UF} / \mathrm{kg}$ de matière sèche. Par ailleurs, les animaux de chaque couple ont ingéré la même quantité d'un foin de luzerne sous forme longue (quantité ajustée chaque jour en fonction de la plus faible consommation de la veille) et tous ont pu consommer la paille de leur litière.

Les animaux ont été alimentés individuellement. Ils ont été abattus par bloc de quatre (un couple de boufs et un couple de taureaux), lorsque l'état d'engraissement des taureaux recevant les betteraves a été jugé satisfaisant; la durée de la période expérimentale a été en moyenne de Io7 \pm I 5 jours.

Pour caractériser la proportion respective de muscles et de dépôts adipeux, nous avons disséqué la i I $^{\mathrm{e}}$ côte prélevée sur chaque demi-carcasse (Y. GEAy, C. Béranger, r969).

\section{RÉSULTATS ET DISCUSSION}

\section{Influence de la castration}

Les bœufs ont eu un gain de poids vif total significativement plus faible ( 24 p. roo) que celui des taureaux au cours des Io7 jours d'engraissement (tabl. I, fig. 3). Cependant, ils ont ingéré une quantité de matière sèche non significativement différente. Par suite, leur efficacité alimentaire a été moins bonne (23 p. roo) ( $\mathrm{P}<\mathrm{o}, \mathrm{or})$. A l'abattage, les taureaux ont eu des carcasses significativement plus lourdes et moins riches en dépôts adipeux ( $\mathrm{P}<0, \mathrm{or})$ que les bœufs (tabl. 2), tout en présentant un état d'engraissement satisfaisant. Les taureaux, qui pesaient $12,6 \mathrm{~kg}$ de plus que les bœufs au début de l'engraissement, ont fourni $26,4 \mathrm{~kg}$ de carcasse en plus. En revanche, la proportion d'avant dans la carcasse a été supérieure de 3 , I points à celle des bœufs (tabl. 2). Les taureaux produisent, à un coût énergétique moindre, une plus grande quantité d'une viande moins riche en graisse. Nous n'avons malheureusement pas pu étudier les qualités organoleptiques de cette viande. Cependant P. R. Woodhams et J. S. Trower (I965) ont comparé la qualité de la viande de bceufs castrés à ro semaines et abattus à 22-23 mois à celle des taureaux abattus au même âge et élevés dans les mêmes conditions. Ces auteurs n'ont pas mis en évidence de différences significatives en ce qui concerne la succulence et l'arôme, seule la tendreté de la viande de bœuf était légèrement supérieure à celle de la viande de taureau $(P<O, I)$. 


\begin{tabular}{|c|c|c|c|c|c|c|c|c|}
\hline \multirow{9}{*}{ 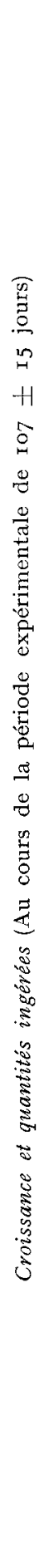 } & \multirow{2}{*}{ 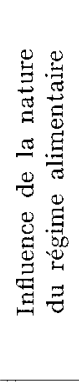 } & 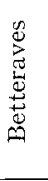 & \multicolumn{2}{|l|}{ 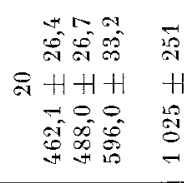 } & 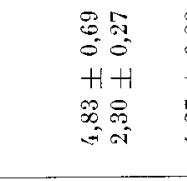 & 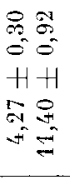 & 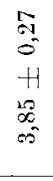 & 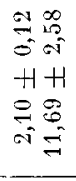 \\
\hline & & $\begin{array}{l}\stackrel{0}{0} \\
\stackrel{5}{5}\end{array}$ & 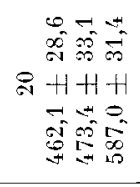 & 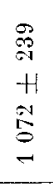 & 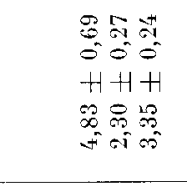 & 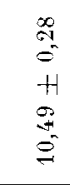 & $\begin{array}{l}\infty \\
8 \\
0 \\
0 \\
11 \\
11 \\
0 \\
0 \\
\infty \\
\infty\end{array}$ & 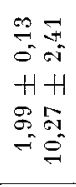 \\
\hline & 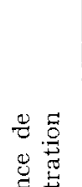 & $\begin{array}{l}\stackrel{n}{7} \\
\stackrel{8}{3}\end{array}$ & 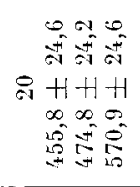 & $\begin{array}{l}\stackrel{\Delta}{\Delta} \\
+1 \\
0 \\
\sigma\end{array}$ & 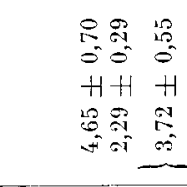 & 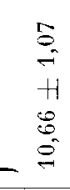 & $\begin{array}{l}\infty \\
01 \\
0 \\
+1 \\
H \\
10 \\
0 \\
\infty\end{array}$ & 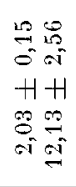 \\
\hline & 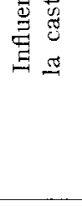 & 总 & 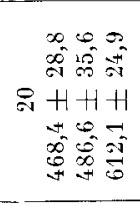 & 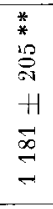 & 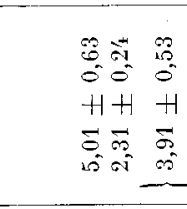 & $\begin{array}{l}8 \\
8 \\
+1 \\
0 \\
0 \\
= \\
=\end{array}$ & $\begin{array}{l}\text { s. } \\
0 \\
0 \\
+1 \\
+1 \\
0 \\
0\end{array}$ & 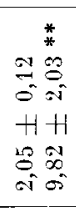 \\
\hline & 光 & 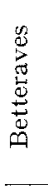 & 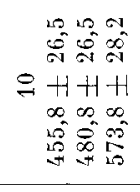 & 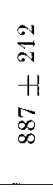 & 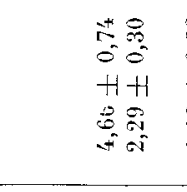 & $\begin{array}{l}0 \\
0 \\
0 \\
0+1 \\
+1+1 \\
\infty \\
\rightarrow= \\
=\end{array}$ & 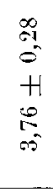 & 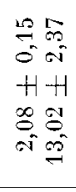 \\
\hline & p & $\begin{array}{l}\stackrel{5}{\infty} \\
\stackrel{\infty}{0}\end{array}$ & 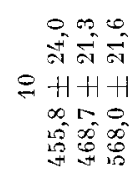 & 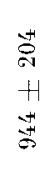 & 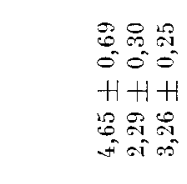 & 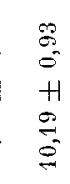 & 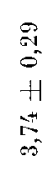 & 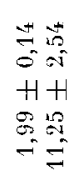 \\
\hline & $\stackrel{\sharp}{\exists}$ & 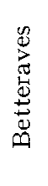 & 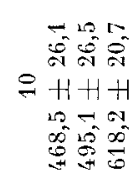 & 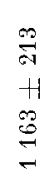 & 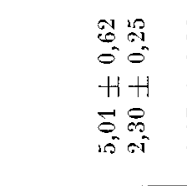 & 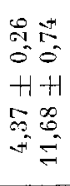 & 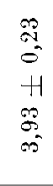 & $\begin{array}{l}8= \\
8=-1 \\
0=0 \\
H+1 \\
=0 \\
=0\end{array}$ \\
\hline & $\stackrel{\Xi}{\vec{G}}$ & 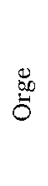 & 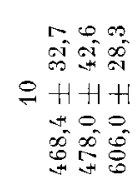 & $\begin{array}{l}\stackrel{2}{8} \\
+1 \\
\stackrel{H}{9} \\
\stackrel{9}{二}\end{array}$ & 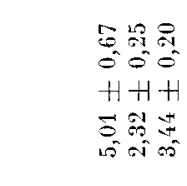 & 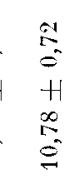 & 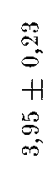 & 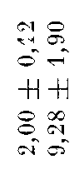 \\
\hline & & & 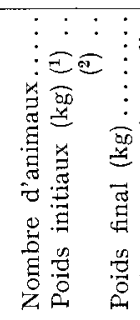 & 窇: & 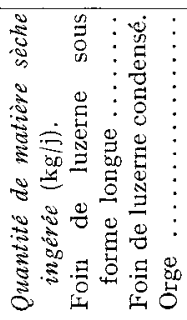 & 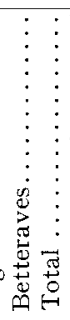 & & 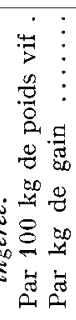 \\
\hline
\end{tabular}

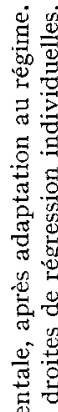

ง.

은 ते

횽 $\pi$ 恋济

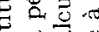
의 종

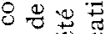

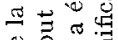

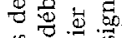
호률 ․ㅡㅇ $\stackrel{\Xi}{\Xi}$ 过 䨌需空 承造

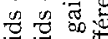

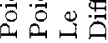
Е可和 
L'influence de la castration à l'âge de to mois s'est donc remarquablement manifestée durant la période d'engraissement qui a commencé ro mois plus tard, cela chez des animaux qui étaient pourtant de race précoce et de poids très peu différents à 20 mois. Cependant, ce phénomène aurait peut-être été moins sensible si les animaux avaient reçu dans l'intervalle une ration leur permettant d'extérioriser plus tôt de telles différences.

Poids, rendement, conformation et ét

Poids vif vide $(\mathrm{kg})$

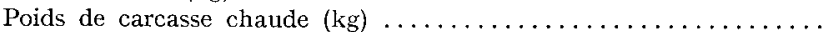

Poids de carcasse chaude

Poids vif vide

\begin{tabular}{|c|}
\hline 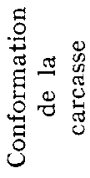 \\
\hline 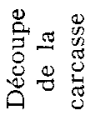 \\
\hline
\end{tabular}

\begin{tabular}{|c|}
\hline $\begin{array}{l}\frac{\text { Poids de carcasse froide }}{\text { Longueur totale }}(\mathrm{kg} / \mathrm{cm}) \ldots \ldots \ldots \\
\frac{\text { Épaisseur de cuisse }}{\text { Jarret-Symphyse }} \times 100 \ldots \ldots \ldots \ldots\end{array}$ \\
\hline $\begin{array}{l}\frac{\text { Poids du "pan traité " }}{\text { Poids de carcasse }} \times 100 \ldots \ldots \ldots \ldots \ldots \\
\frac{\text { Poids de l'épaule et du collier }}{\text { Poids de carcasse }} \times 100 \ldots \ldots \ldots\end{array}$ \\
\hline $\begin{array}{l}\text { Dépôts adipeux périrénal et précural (p. } 100 \\
\text { du poids de carcasse) } \ldots \ldots \ldots \ldots \ldots \ldots \ldots \ldots \\
\text { Proportion dans la " } 11 \text { e côte } " \text { : } \\
\text { - Muscle (p. } 100) \ldots \ldots \ldots \ldots \ldots \ldots \ldots \ldots \ldots \ldots \\
\text { - Dépôts adipeux }(p .100) \ldots \ldots \ldots \ldots \ldots\end{array}$ \\
\hline
\end{tabular}

Taureaux

\begin{tabular}{|c|c|}
\hline Orge & Betteraves \\
\hline $\begin{array}{l}516,1 \pm 24,0 \\
341,5 \pm 18,3\end{array}$ & $\begin{array}{l}519,8 \pm 11,7 \\
345,3 \pm 10,9\end{array}$ \\
\hline $66,2 \pm 1,0$ & $66,4 \pm 1,3$ \\
\hline $2,50 \pm 0,08$ & $2,52 \pm 0,08$ \\
\hline $31,5 \pm 0,8$ & $31,9 \pm 1,0$ \\
\hline $48,3 \pm 1,1$ & $48,8 \pm 1,0$ \\
\hline $47,8 \pm 1,5$ & $47,8 \pm 1,0$ \\
\hline $1,71 \pm 0,49$ & $1,63 \pm 0,47$ \\
\hline $66,6 \pm 3,1$ & $68,1 \pm 2,1$ \\
\hline $15,1 \pm 2,1$ & $14,5 \pm 2,5$ \\
\hline
\end{tabular}

\section{Influence de la nature du régime alimentaire}

Au cours de la période expérimentale, les animaux recevant l'orge ont bien ingéré la même quantité d'énergie nette (UF) que leurs homologues recevant les betteraves (50 p. roo de la ration), ainsi que le prévoyait le protocole expérimental (tabl. I). Ils ont eu le même crốt journalier et ont fourni des carcasses de poids et de composition non significativement différentes (tabl. I et 2 ; fig. I, 2 et 4). La nature de la ration n'a donc pas modifié le gain de poids vif et la composition des carcasses de façon notable.

La valeur énergétique de o,9 UF attribuée aux betteraves pour l'engraissement se trouve donc confirmée. En revanche, elle serait trop élevée pour la production laitière selon les observations de Journer (résultats non publiés). Cependant, les bœufs recevant les betteraves ont fourni des carcasses légèrement plus grasses que leurs homologues recevant l'orge. Cette tendance n'est cependant pas significative; elle le serait peut-être devenue si le nombre d'animaux avait été plus mportant, leur hétérogénéité moins grande et si la proportion d'orge et de betteraves avait été plus élevée (respectivement 32 et 37 p. Ioo de la matière sèche de la ration). 
En conclusion, dans le cadre d'une production de viande, à partir d'animaux abattus à 2 ans, la castration a entraîné une augmentation de la proportion des dépôts adipeux aux dépens de celle des muscles et une diminution du gain de poids vif. En revanche, la nature du régime alimentaire n'a pas permis de modifier significativement la composition des carcasses des animaux. En particulier, il n'a pas été possible de diminuer l'état d'engraissement des carcasses des bœufs et

engraissement des carcasses

\begin{tabular}{|c|c|c|c|c|c|}
\hline \multicolumn{2}{|c|}{ Bœufs } & \multicolumn{2}{|c|}{ Influence de la castration } & \multicolumn{2}{|c|}{$\begin{array}{l}\text { Influence de la nature } \\
\text { du régime alimentaire }\end{array}$} \\
\hline Orge & Betteraves & Taureaux & $B$ ixufs & Orge & Betteraves \\
\hline $\begin{array}{l}477,7 \pm 29,7 \\
313,3 \pm 21,1\end{array}$ & $\begin{array}{l}488,1 \pm 21,3 \\
320,7 \pm 13,6\end{array}$ & $\begin{array}{l}518,0^{* *} \pm 18,5 \\
343,4 * * \pm 1 / 4,8\end{array}$ & $\begin{array}{l}482,9 \pm 25,7 \\
317,0 \pm 17,7\end{array}$ & $\begin{array}{l}496,9 \pm 32,8 \\
327,4 \pm 24,1\end{array}$ & $\begin{array}{l}504,0 \pm 23,3 \\
333,0 \pm 17,4\end{array}$ \\
\hline $65,6 \pm 1,6$ & $65,7 \pm 0,9$ & $66,3 \pm 1,1$ & $65,7 \pm 1,3$ & $65,9 \pm 1,3$ & $66,1 \pm 1,2$ \\
\hline $2,31 \pm 0,13$ & $2,38 \pm 0,08$ & $2,51 * * \pm 0,08$ & $2,35 \pm 0,11$ & $2,40 \pm 0,14$ & $2,45 \pm 0,11$ \\
\hline $30,9 \pm 1,4$ & $31,1 \pm 1,1$ & $31,8 * * \pm 0,9$ & $31,0 \pm 1,3$ & $31,2 \pm 1,2$ & $31,5 \pm 1,1$ \\
\hline $50,2 \pm 1,6$ & $49,8 \pm 1,1$ & $48,5^{* *} \pm 1,1$ & $50,0 \pm 1,3$ & $49,2 \pm 1,7$ & $49,3 \pm 1,2$ \\
\hline $45,0 \pm 0,6$ & $4,4,4 \pm 0,6$ & $47,8^{* *} \pm 1,2$ & $44,7 \pm 0,6$ & $46,4 \pm 1,8$ & $46,0 \pm 1,9$ \\
\hline $2,93 \pm 0,98$ & $3,46 \pm 0,74$ & $1,67^{* *} \pm 0,47$ & $3,19 \pm 0,89$ & $2,32 \pm 0,98$ & $2,55 \pm 1,12$ \\
\hline $63,5 \pm 4,1$ & $60,9 \pm 5,0$ & $67,3^{* *} \pm 2,7$ & $62,2 \pm 4,6$ & $65,0 \pm 3,9$ & $64,3 \pm 5,3$ \\
\hline $18,8 \pm 3,9$ & $22,0 \pm 3,2$ & $14,8^{* *} \pm 2,2$ & $20,4 \pm 3,8$ & $17,0 \pm 3,6$ & $18,4 \pm 4,7$ \\
\hline
\end{tabular}

d'augmenter leur gain de poids vif par un apport de saccharose dans la ration en remplacement de l'amidon de l'orge.

Reçu pour publication en février 1971.

\section{SUMMARY}

THE GROWTH AND QUALITY OF THE CARCASSES OF CATTLE SLAUGHTERED AT 24 MONTHS AS INFLUENCED BY CASTRATION AND THE NATURE OF THE GLUCIDES IN THE DIE'T

According to a factorial design, we compared, on the one hand, the performances of 20 steers with those of 20 bulls fattened between 20 and 24 months and, on the other hand, two diets which should supply the same amount of net energy, either in the form of fooder beets or in the form 


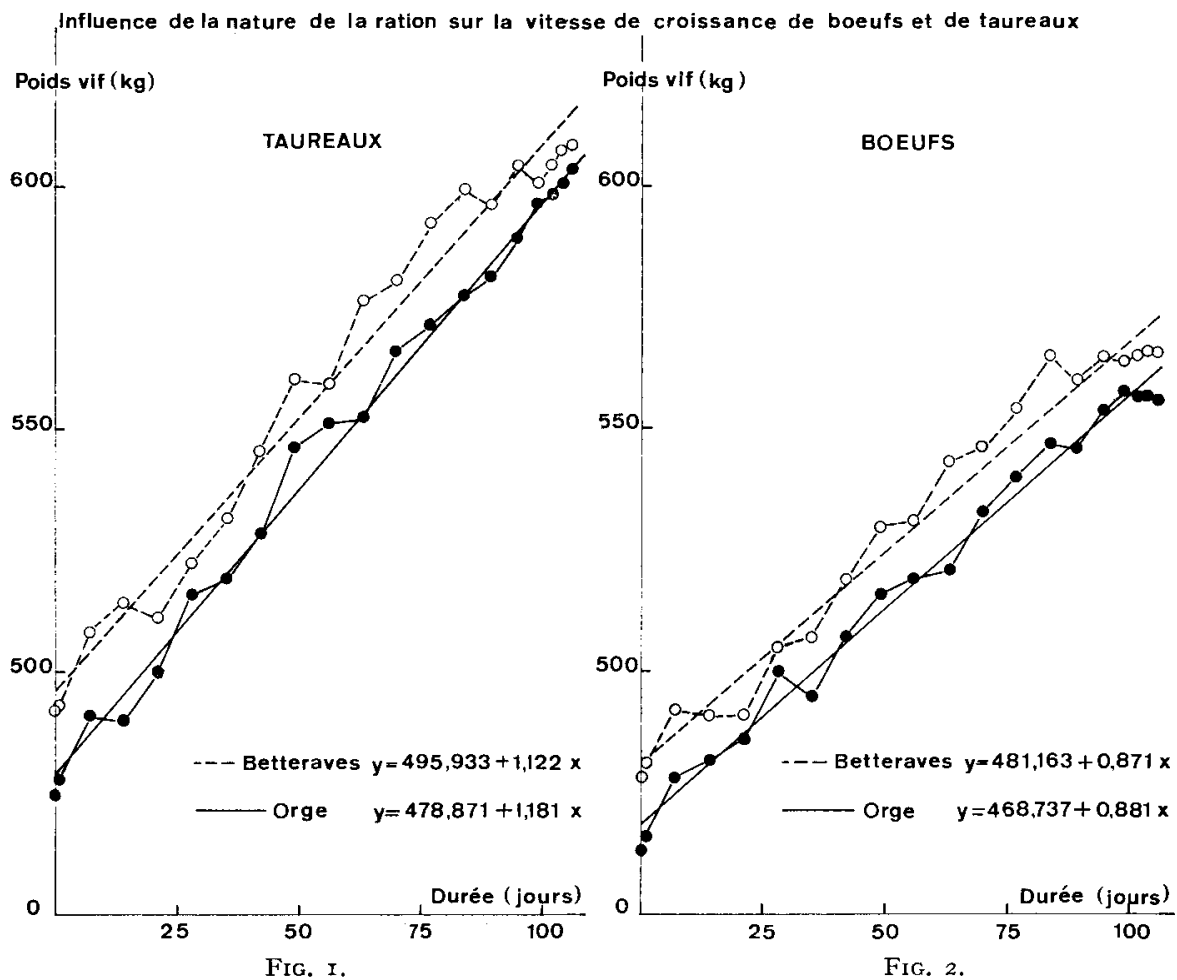

FIG. I.

Poids vif (kg)

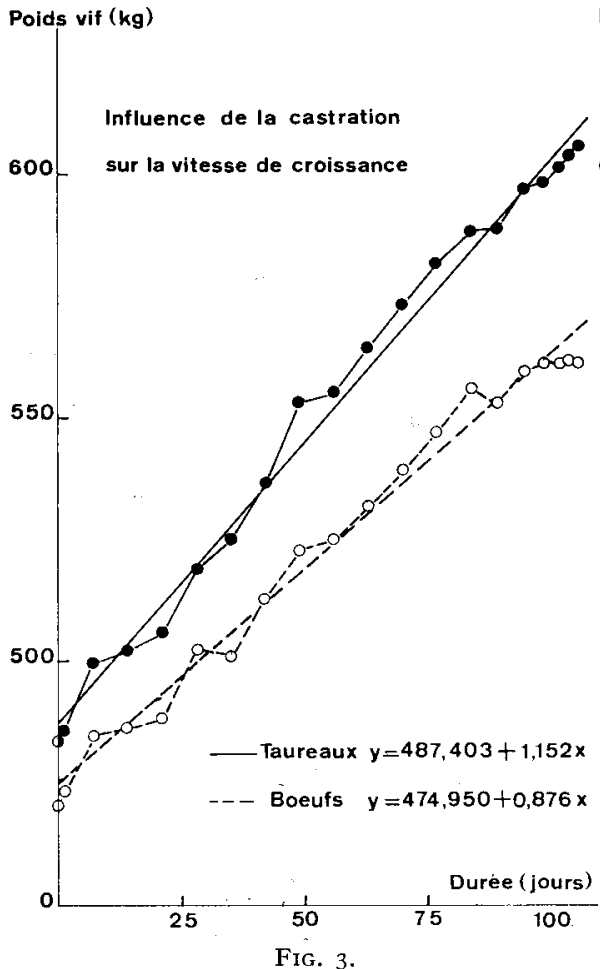

Influence de la nature du regime 600. alimentaire sur la vitesse de croissance

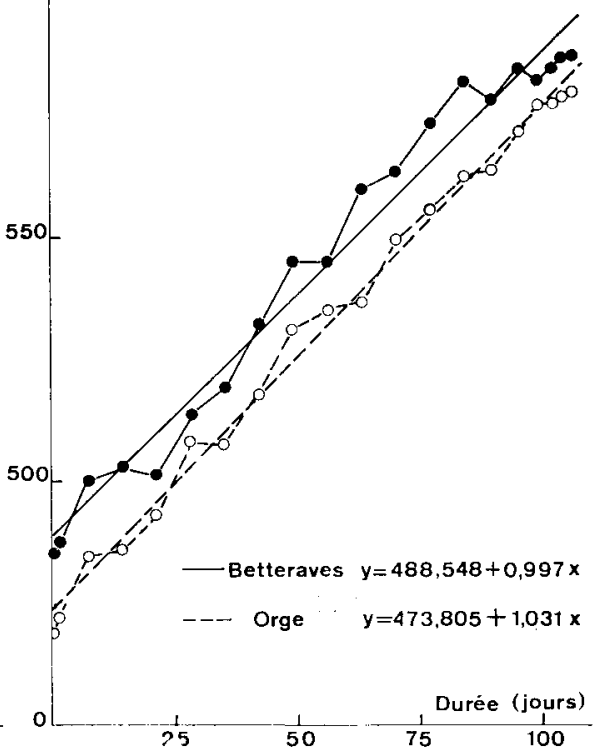

FIG. 4. 
of barley. The castration decreased the live weight gain (244 p. 100) and the weight of thecarcasses ( $8 \mathrm{p}$. Ioo), but the proportion of fat was increased $(38 \mathrm{p}$. Ioo). On the other hand, even when substituting the barley starch of the ration by the saccharose of the beets it was not possible neither to decrease the fatness of the steers nor to increase their live weight gain.

\section{RÉFÉRENCES BIBLIOGRAPHIQUES}

Béranger C., Petit M., I97o. Production de jeunes bovins de boucherie à partir d'herbe. C. R. des journées d'études sur la production des jeunes bovins. S. E. I. (sous presse).

BRÄNNÄNG E., 1969. The raising of bulls for beef in Sweden in Rhodes D. N. Meat production from entire animals, p. I73, Churchill led.

GEAy Y., Béranger C., I 969 . Influence de la proportion de céréales dans la ration sur l'état d'engraissement des carcasses de taurillons de 5 mois. Ann. Zootech., 18, 79-8r.

GEAY Y., BÉRANGer C., I 969 . Estimation de la composition de la carcasse de jeunes bovins à partir de la composition d'un morceau monocostal au niveau de la II ${ }^{\mathrm{e}}$ côte. A $\mathrm{nn}$. Zootech., 18, 65-77.

Klosterman E. W., Kunkle I. E., Gerlaugh P., Cahill V. R., i954. The effect of age of castration upon rate and economy of gain and carcass quality of beef calves. J. Anim. Sci., 13, 817-825.

Richter K., Cranz K. L., Schmidt K. H., rg60 $a$. Mastversuche mit jungbullen und junguchsen n⿳ ${ }^{\circ}$ Züchtungskunde, 32 (5), 2 I 7-230.

Richter K., Cranz K. L., Schmidt K. H., i960 b. Mastversuche mit jungbullen und junguchsen $\mathrm{n}^{\circ}$ 2. Züchtungskunde, $32(5), 560-574$.

Richter K., Cranz K. L., Schmidt K. H., I96r. Mastversuche mit jungbullen und junguchsen n ${ }^{\circ}$ 3. Züchtungskunde, 33 (10), 493-5ro.

Turton J. D., I969. The effect of castration on meat production for cattle, sheep and pigs In Rhodes D. N. Meat production from entive animals, p. 149, Churchill Ed.

Woodhams Pamela R., Trower Susan J., 1965. Palatability characteristics of rib-steaks from $A$ berdeen Angus steers and bulls. N.Z.J. Agric. Res., 8, 921-926. 